\title{
La secrète revendication d'une sexualité féminine : les chants du marcanda chantés par des femmes songhay-zarma d'origine captive (Niger)
}

\section{Sandra Bornand}

\section{(2) OpenEdition}

Journals

Édition électronique

URL : https://journals.openedition.org/clo/1477

DOI : $10.4000 /$ clo. 1477

ISSN : 2266-1816

Éditeur

INALCO

Édition imprimée

Date de publication : 1 janvier 2012

Pagination : 79-115

ISBN : 978-2-85831-206-1

ISSN : 0396-891X

Référence électronique

Sandra Bornand, «La secrète revendication d'une sexualité féminine : les chants du marcanda chantés par des femmes songhay-zarma d'origine captive (Niger) », Cahiers de littérature orale [En ligne], 71 | 2012, mis en ligne le 17 avril 2014, consulté le 01 juillet 2021. URL : http://journals.openedition.org/ clo/1477; DOI : https://doi.org/10.4000/clo.1477

Ce document a été généré automatiquement le 1 juillet 2021.

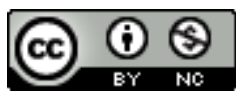

Cahiers de littérature orale est mis à disposition selon les termes de la Licence Creative Commons Attribution - Pas d'Utilisation Commerciale 4.0 International. 


\section{La secrète revendication d'une sexualité féminine : les chants du marcanda chantés par des femmes songhay-zarma d'origine captive (Niger)}

\section{Sandra Bornand}

\section{Introduction}

1 Au Niger, en région songhay comme zarma ${ }^{1}$, la polygamie est courante. C'est dans ce contexte qu'a lieu un rituel, le marcanda, organisé pour la dernière épousée d'un homme qui s'apprête à se remarier. À la nuit tombée, après la bénédiction du mariage, les proches de cette dernière (toutes des femmes mariées) s'affrontent - de manière ludique - lors d'une joute verbale d'insultes à laquelle ne participe pas leur hôtesse, qui ne peut quitter sa chambre: les premières épousées (les "grandes épouses») et les suivantes ${ }^{2}$ («les petites épouses ») échangent des insultes ritualisées, en dansant et en chantant (Bornand, 2002, 2010). Parfois, ce sont des femmes d'origine captive qui viennent chanter ; ces dernières évoquent, dans un répertoire qui leur est propre, et en l'absence des hommes, la sexualité, ses plaisirs, ses souffrances ainsi que ses déviances.

2 Cet article propose une analyse de ces chants obscènes enregistrés en 2003 à Niamey, capitale du Niger ${ }^{3}$, et montrera comment les chanteuses abordent un sujet aussi tabou pour la société songhay-zarma, oscillant en permanence entre l'explicite et le non-dit. Dans un deuxième temps, on s'interrogera sur les objectifs de ces chants : sont-ils un moyen de construire la représentation d'une identité féminine et des relations avec les hommes différente de celle véhiculée par l'idéologie dominante ou une simple étape du marcanda ? Visant en effet à garantir une paix sociale et familiale menacée par l'arrivée 
d'une nouvelle épouse dans le foyer, ce rituel peut finalement s'avérer " consensuel », au-delà des apparences.

\section{Une parole décomplexée?}

3 La situation de communication ${ }^{4}$ semble plaider en faveur d'une parole assumée : d'une part, parce que les chants du marcanda sont chantés en présence d'autres femmes mariées et d'autre part, parce qu'ils participent d'une énonciation collective: non seulement les chanteuses s'interpellent et se répondent tout au long de la performance, mais elles s'inscrivent dans une continuité temporelle relevant de la tradition orale. Leur prestation est ainsi, en quelque sorte, instituée et « reproduite » dans les mêmes occasions depuis des générations.

4 Toutefois, l'origine sociale des chanteuses ainsi que l'auditoire et le lieu de la performance demandent de nuancer notre propos. En effet, si les énonciataires du marcanda peuvent en général être des femmes de toute extraction (y compris d'origine libre, ou «noble » en français nigérien), les chanteuses de ce répertoire particulier ne peuvent être que d'origine captive. Et ceci n'est pas un détail, car il se dit en régions zarma comme songhay que « le captif ne connaît pas la honte » (banna si haawi ${ }^{5}$ bay), au contraire de l'homme libre qui se doit de manifester une retenue et une pudeur en toutes circonstances. Aussi faire chanter les femmes d'origine captive est-il un moyen de contourner les obstacles à l'évocation d'une sexualité parfois débridée sans contrevenir aux règles de la société, faites par et pour les hommes libres. Cela d'autant plus que l'«idéologie esclavagiste» - pour reprendre une expression d'olivier de Sardan (1984) - reste prégnante aujourd'hui encore en région songhay-zarma (alors même qu'il n'y a plus d'esclavage) et que ces chanteuses à la parole licencieuse se situent doublement au bas de l'échelle sociale : comme femmes, mais aussi et surtout comme descendantes de captives.

5 Une autre stratégie de contournement, du point de vue de la situation de communication, est le choix du lieu de la performance. Lors d'un marcanda où n'est pas évoquée la sexualité, les chanteuses s'installent généralement dans la concession devant la maison de celle qui perd son rang de dernière épousée (Bornand, 2002, 2010). Mais, dans le cas qui nous intéresse ici, elles chantent à l'intérieur de la maison en présence uniquement des femmes mariées, afin que ni les enfants, ni les jeunes filles, ni les hommes n'entendent ce qui s'y dit.

6 Pourtant, aujourd'hui, malgré les stratégies mises en place, les occasions de chanter deviennent toujours plus rares: soit parce que les hommes - à qui reviennent l'autorisation et le financement du rituel - avertissent de moins en moins leurs épouses de leur nouveau mariage (contrairement à ce que l'islam prescrit), car ils craignent les sorts qu'elles pourraient leur jeter. Elles ne peuvent alors plus organiser la cérémonie ; soit parce que lorsqu'ils les préviennent et leur offrent le repas, ils interdisent les joutes verbales et les chants, par crainte de débordements ou au prétexte que l'islam interdit l'insulte; soit enfin parce que certains époux autorisent que le marcanda se déroule normalement, mais interdisent les chants paillards toujours au nom de la religion musulmane.

7 Ainsi, alors que je connaissais très bien les chanteuses et qu'elles m'avertissaient des différentes cérémonies auxquelles elles étaient conviées, je n'ai jamais pu enregistrer de performances en situation de communication rituelle, celles-ci ayant été 
systématiquement interdites ou décommandées au dernier moment. Et, depuis l'enregistrement réalisé à Niamey et présenté ici, ces femmes m'ont rapporté qu'elles n'avaient plus jamais eu l'occasion de chanter ces chants paillards. Ceci explique pourquoi l'enregistrement analysé a été réalisé sur proposition des chanteuses, mais en situation de communication « artificielle ", loin des oreilles et des regards masculins ${ }^{6}$.

\section{Une énonciation explicite}

D'un point de vue discursif, la parole assumée passe par le dépassement explicite du tabou. Dans leurs chants, les chanteuses énumèrent et décrivent explicitement, voire avec une certaine gourmandise, les organes génitaux, le plaisir et l'acte sexuel. Elles y racontent aussi des anecdotes où les protagonistes rompent un tabou social comme ce beau-père couchant avec sa belle-fille ou ce marabout convoitant la femme d'autrui. Les chanteuses expriment ainsi l'absence de maîtrise de soi par les mots, mais aussi par leurs danses qui simulent parfois l'acte sexuel; toute leur prestation est donc en opposition avec l'idéal social et moral de "pudeur» qui prédomine en permanence dans la société songhay-zarma.

\section{Nomination et description}

9 La nomination et la description, à travers lesquelles les chanteuses se construisent une position d'expertes des choses du sexe, relèvent des procédures où elles assument pleinement et explicitement leurs propos.

\section{La nomination}

10 J'ai enquêté sur le vocabulaire d'ordre sexuel auprès d'hommes et de femmes de toutes origines sociales. Bien que je n'aie mené mes entretiens qu'avec des personnes que je connaissais au préalable et que j'aie le plus souvent délégué à un homme le soin de s'entretenir avec les hommes d'origine libre, ces échanges se sont révélés difficiles, car la sexualité reste taboue dans la vie courante. Tous mes interlocuteurs se sont montrés mal à l'aise à l'exception des chanteuses et d'un joueur de tambour d'aisselle.

11 Sur la base de discussions individuelles informelles, j'ai demandé à ce que l'on classe les termes sexuels connus du moins au plus honteux. La classification ainsi obtenue est relativement uniforme et on ne décèle pas de différence significative liée au genre ou à l'extraction sociale. Sur la base de ces résultats, j'ai tenté, dans ma traduction, de chercher des équivalents en français, les mieux à même de rendre les connotations et les représentations des dénominations. On découvre par exemple que le suffixe -ize (fils, petit) associé à des termes sexuels connote péjorativement ceux-ci, ce qui n'est pas $\mathrm{du}$ tout le cas pour d'autres paradigmes ${ }^{7}$. Ainsi dafayize (litt. "l'enfant du sexe féminin », qui désigne le clitoris et que je traduis ici par «berlingot » pour retranscrire la connotation impudique du terme ${ }^{8}$ ) parait plus indécent que dafe (« con»).

Alors qu'au quotidien (à la rare exception de jurons tels que na dafe, " con de la mère ", baaba bande " bite du père $»^{9}$ ) les organes génitaux sont pudiquement désignés par le terme générique de "chose» (hay), les chanteuses énumèrent ici, avec une joie certaine, les parties du sexe féminin et masculin. Plus encore, elles y multiplient les registres linguistiques, passant tantôt du familier au vulgaire. Aussi parlent-elles de bas-ventre (koje) $)^{10}$, de con (dafe), de la chatte (koosi), de la foufounette (natte), de la 
moule (kuna), du berlingot (dafayize) ou encore du clito (kunayize) pour le sexe féminin. Elles citent la bite (bande, haafe), la queue (hanji) ou les couilles (fooru, fooriize) pour le sexe masculin.

Concernant l'acte sexuel, si le jasare (griot généalogiste et historien) le qualifie, dans ses récits, par le terme - moins vulgaire - de goyandi (litt. " faire travailler ») qui signifie dans ce cadre "besogner", les chanteuses, elles, varieront les désignations et diront kaaru («monter, chevaucher») voire, plus vulgairement, joko («baiser»), Jaajin (« troncher ») :

Dafe day ga ti koyize kan i ga a gar ganda ga a noko kubay ra

C'est vraiment le con qui est prince, lui qu'on trouve en bas pour le baiser dans

l'obscurité!

(Chant 6, v. 5)

Dafe day ga ti koyize kan i ga a gar ganda ga a jaajin kubay ra

C'est vraiment le con qui est prince, lui qu'on trouve en bas pour le troncher dans

l'obscurité !

(Chant 6, v. 10)

Elles n'hésitent en outre pas à parler de fellation (haafu sunsumyan: littéralement «sucer la bite »), une pratique considérée comme particulièrement immorale.

L'intensité et le paroxysme de la jouissance féminine se dégagent des verbes dénotant une action vive, voire violente; une action exercée sur un organe sexuel placé en complément d'objet :

1) Ay ga hanna ga sinji no / I ma ay dafayizo kar ga say

Je resterai assise toute la nuit / Pour qu'on déchiquette mon berlingot.

(Chant 12, v. 3-4)

2) Ay ga hanna ga sinji no ay ga hanna ga sinji no / I ma duya ${ }^{11}$ day ga a bogu-boga ${ }^{12}$

Je resterai assiste toute la nuit, je resterai assise toute la nuit / Jusqu'à ce qu'on le

casse vraiment en petits morceaux.

(Chant 12, v. 22-23)

Elles ne font pas que nommer ou décrire, mais exposent aussi les conséquences $d u$ dépassement du tabou de la sexualité. C'est le cas par exemple quand une soliste invite tout un chacun à taire certains événements honteux, tout en se permettant paradoxalement - de les raconter par la suite. C'est d'ailleurs par ce chant que les femmes commencent en général les performances liées à ce répertoire (même si ce n'est pas le cas ici) comme pour se dédouaner de ce qu'elles vont dire par la suite :

1) Haawo si deede

La honte ne se raconte pas.

(Chant 11, v. 11)

2) Tu ma ga deede a haawu

Qui entend et rapporte honte à lui !

(Chant 21, v. 16)

17 La soliste semble, une fois le tabou brisé, se complaire dans le dépassement de l'interdit, répétant inlassablement - comme par gourmandise - les termes désignant le sexe masculin :

Iri mo kan ga bay ma ne bande bande bande bande bande bande [...]

Et nous qui connaissons disons 'bite' 'bite' 'bite' 'bite' 'bite' 'bite' [...].

(Chant 8, v. 13)

18 Remarquons que l'emploi de la première personne du pluriel crée une connivence avec les autres femmes mariées avec qui elle partage la position d'experte, et qu'elle crée 
une opposition avec la jeune mariée, novice dans ce domaine au point de ne pas distinguer - disent-elles - le sexe de l'homme d'une racine de manioc :

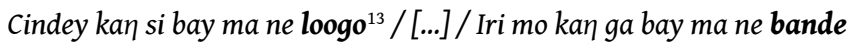

Certaines qui ne savent pas disent que c'est du manioc / [...] / Et nous qui savons disons que c'est une bite.

(Chant 8, v. 9)

Et la transgression n'est pas seulement liée aux mots. Montrer que l'on maîtrise les choses du sexe et évoquer son plaisir sont eux aussi des manquements à la morale sociale; on apprend d'ailleurs à la jeune mariée (dans les rares cas où on lui en parle) à ne pas montrer son plaisir lors de l'acte sexuel. Or les solistes - comme enivrées par leurs propos (et leurs pensées?) - poussent toujours plus loin la provocation et vont jusqu'à mettre en scène un marabout convoitant la femme d'autrui alors qu'il est en train d'enseigner le Coran à ses élèves !

\section{La description}

La nomination des organes génitaux est souvent accompagnée d'épithètes dont la connotation, positive ou négative, permet de dégager un idéal des sexes féminin et masculin.

Aussi le premier (d'abord nommé par son euphémisme hay « chose », puis par son nom dafe « con ») se doit-il d'être grand pour être mieux pénétré :

Ay manti hay kayna / Ay manti koosi kayna

Je ne suis pas une petite chose / Je ne suis pas une petite chatte.

(Chant 4, v. 4-5)

L'association, par comparaison ou métaphore, à des objets du quotidien, tous récipients de forme concave, permet de louer sa profondeur: la calebasse (gaasu), le récipient (koppiize), mais aussi - lorsque la femme n'est plus vierge - l'emplacement (gurbo) ou le trou (gatambo). Signalons par ailleurs que ces objets sont souvent liés à la nourriture et que les femmes établissent, de fait, métaphoriquement ou comparativement, un lien entre sexe et nourriture. Un lien bien réel, puisque la coépouse qui prépare le repas est celle qui passera la nuit avec son mari.

S'agissant de décrire le sexe masculin, ces associations par comparaison ou métaphore renvoient assez classiquement à des végétaux ou à des objets de forme phalliques issus du quotidien. Ainsi le pénis est-il pilon (hinji), fouet (fifirji ${ }^{14}$ ), fourche (ganji), poutre (warra), os (biri) ou manioc (roogo). Outre les caractéristiques formelles, le sexe masculin est « long » «beau et bon » (boori), mais aussi « chaud» (dungu), la sensation de chaleur caractérisant le plaisir féminin :

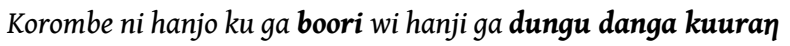

Korombé ! ta queue bien longue et belle est-il possible qu'une queue soit aussi

chaude qu'un courant électrique ?

(Chant 16, v. 17)

Ce sexe se doit - pour donner du plaisir à la femme - d'être long, élastique, mais aussi fin, les chanteuses raillant le petit, le tordu ou le gros:

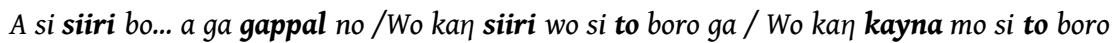
ga / Wo kan warga mo ga hayfun no / Kala day birkitik / [...] / A jeeneewa ${ }^{15}$

Ce n'est pas vraiment qu'elle se tord... c'est qu'elle s'aplatit ${ }^{16} /$ celle qui est tordue ne t'atteint pas / Et celle qui est petite ne t'atteint pas / Et celle qui est grosse est paresseuse / Il faut la géante / [...] / elle se met en érection.

(Chant 7, v. 14-19)
} 
Les relations sexuelles sont très peu décrites explicitement ; les chanteuses préfèrent la métaphore filée, comme dans le vingt-septième chant, où les termes baata («botte de paille sèche ») et boogu (" travail collectif»), s'ils renvoient dans un premier temps à la nature, sont très vite associés à la sexualité par la contamination qu'opère le cinquième vers :

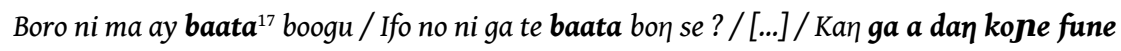

ra

Une telle, viens-moi en aide avec une botte de paille sèche / Qu'est-ce qu'on fait avec une botte de paille sèche ? / [...] / [C'est] pour la mettre dans le trou du bas-ventre.

(Chant 27, v. 1-5)

seule comparaison étant celle de «l'enclume [résistant] au forgeron». Cette comparaison implique l'idée de coups portés par une masse, à laquelle le sexe de la femme résiste pour en retirer du plaisir (cf. les verbes « se tordre » et « frétiller ») :

Mate kan taara mongu zam se / Bora kunayizo ma kuubi wayboro mo hayayizo ma kuubi / Wayboro mo kunayizo ma fittaw.

De la manière dont l'enclume résiste au forgeron / Que le clito de la personne se torde et que la petite chose de la femme se torde / Et que le clito de la femme s'arrache!

(Chant 21, v. 24-26)

On le voit, par la nomination et la description tant des organes que de l'acte, les chanteuses construisent, pour elles-mêmes et les locutrices qu'elles installent dans leurs chants, une position de connaisseuses des choses du sexe; ce qui avalise l'impression qu'elles assument leurs discours sur la sexualité. Cette impression semble être confirmée du point de vue énonciatif par une prise en charge de leurs discours. 


\section{Une prise en charge énonciative}

31 Alliant le geste à la parole, la chanteuse met aussi en scène l'acte sexuel : elle simule parfois par la danse un accouplement et adresse des paroles érotiques à son partenaire virtuel. Cette mise en situation vise un «effet de réel» et exprime publiquement le fantasme :

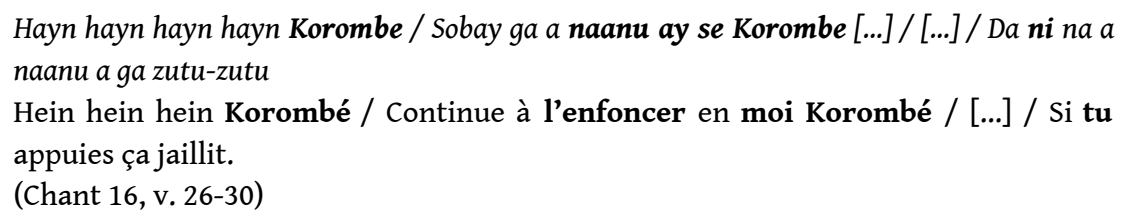

La prise en charge se révèle par l'interjection et dans une énonciation assumée à la première personne du singulier ou du pluriel. Le «je » pose une identité référentielle qui renvoie à l'énonciatrice. Toutefois, dans les chants étudiés, celui-ci est ambigu, car il ne réfère jamais véritablement à la chanteuse, mais au personnage qu'elle fait parler dans un chant de littérature orale ${ }^{19}$. Il y a donc hiatus entre situation de communication et situation d'énonciation.

Mais, même si ce hiatus existe, dire « je », c'est s'associer explicitement au «je » de la locutrice, comme dans l'exemple qui suit:

Ay ga a mottu no ga dan ne wo

Je l'aurais arrachée pour me la mettre ici.

(Chant 8, v. 16)

34 L'association $\mathrm{du}$ " je » et du déictique $n e$ («ici») suivi d'un démonstratif à valeur également déictique $w o^{20}$ ("celui-ci, celle-ci, ceci») crée alors un "effet de réel» (Barthes, 1968). On remarque une opposition entre la femme qui dit «je » et l'homme placé en situation d'objet ${ }^{21}$ : l'énonciatrice nous le présente d'abord réduit à son seul sexe par l'emploi de la synecdoque, puis instrumentalisé et dévolu au seul plaisir de la femme.

La soliste opère ainsi, dans sa description, un renversement des rapports de genre. Alors que, dans les témoignages recueillis, les femmes font référence à la brutalité de l'acte sexuel et à la domination masculine, l'homme venant prendre ce qui lui revient de droit, elle chante ici le plaisir d'une femme et sa supériorité. On retrouve d'ailleurs de nombreuses occurrences de cette inversion:

(soliste 1) [...] bande nda dafe may no ga ti koyize ? / [...] / Dafe day ga ti koyize kaך $i$ ga a gar ganda ga a noko kubay ra [...],

de la bite et le con qui est prince ? / [...] / C'est vraiment le con qui est le chef, lui qu'on trouve en bas pour le baiser dans l'obscurité.

(Chant 6, v. 3-5)

Le "nous ", qui est équivalent à " "je" + un autre ", apparaît ici comme une " personne amplifiée ». Il permet à la chanteuse de s'arroger le soutien de ses paires et ainsi d'élargir la légitimité de son dire. Cette construction d'une communauté favorise l'opposition revendiquée entre les "femmes mariées », dont une représentante énonce ici le "nous", et leurs rivales, ignorantes encore des choses du sexe ${ }^{22}$ - elles sont présumées vierges - dont fait partie la jeune mariée qui arrive. Cette communion dans le «nous", valorisée par l'emploi du verbe « savoir ", passe par l'opposition entre les initiées et les autres, qualifiées péjorativement:

Saamey kan si bay ma ne loogo / To / Iri mo kan ga bay ma ne hanji

Les idiotes qui ne savent pas disent que c'est du manioc / Nous qui savons disons 
que c'est une queue.

(Chant 8, v. 30-31)

Si l'on peut observer, tant du point de vue de la nomination, de la description que de la prise en charge énonciative, une manière d'assumer, voire de revendiquer une sexualité, l'analyse nous porte cependant à nuancer nos propos, car les chanteuses oscillent, au sein des mêmes chants, entre une stratégie de la revendication et une stratégie de la dissimulation.

\section{Du côté du secret et de l'implicite}

Comme l'écrit Kerbrat-Orecchioni $(1998,278)$ : «Bien des choses "ne se disent pas" du moins directement» et les chanteuses, pour des raisons de convenance, recourent souvent à une formulation implicite. Cette dernière sert alors à « conjurer l'existence de certains tabous [...] et [à] ruser avec la loi du silence qui frappe d'interdits certains objets discursifs» (ibid., 277-278). Car quiconque brise un tabou dans la société songhay-zarma risque de voir la honte rejaillir sur lui. Dans un des chants, la soliste fait parler un homme qui a commis un adultère avec sa belle-fille, alors même que son fils était parti gagner sa vie lors d'une migration saisonnière ${ }^{23}$. À travers cette histoire et la stratégie de la dissimulation qu'elle met en scène ( $c f$. le bonnet et le grand boubou qui cachent ce corps qui a fauté), on peut interpréter ces paroles comme un écho ou une mise en abîme des stratégies discursives des solistes qui - en se mettant à l'écart pour chanter, mais aussi en alternant explicite et implicite - brisent un interdit tout en dissimulant leurs propos :

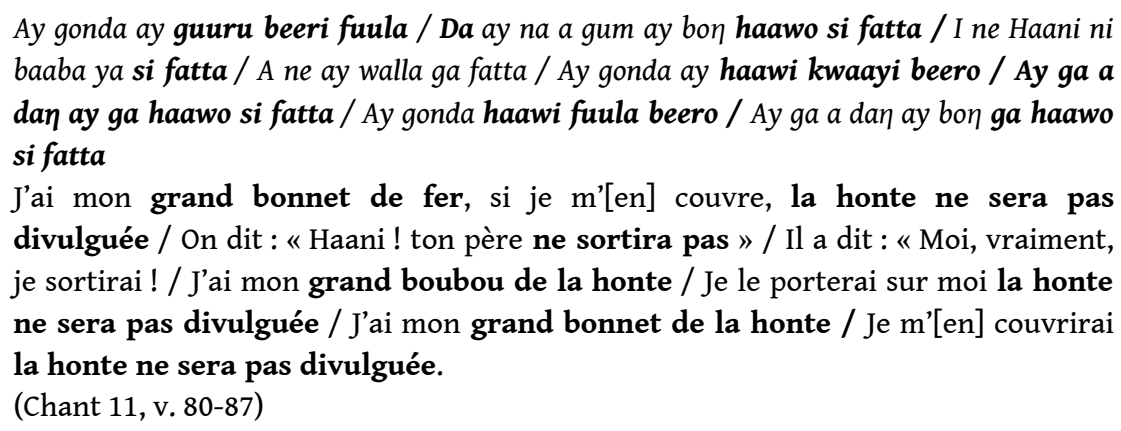

La discrétion, revendiquée ici, est sans cesse transgressée par les chanteuses qui multiplient les anecdotes scabreuses que la bienséance enjoindrait de taire. Le secret se construit donc, tout comme la revendication, à la fois sur la situation de communication (dans une chambre, entre femmes mariées, loin des curieux), sur l'aspect discursif (emploi de tropes, énoncés gnomiques, etc.), mais aussi sur la gestuelle et les regards. Ces éléments paralinguistiques, qui ne seront pas détaillés ici, complètent alors la parole en suggérant ce qu'elle cache ${ }^{24}$.

\section{Les tropes}

Cet informulable est aussi suggéré à l'aide de tropes tels que l'euphémisme, l'ellipse, la métaphore, les onomatopées ou les idéophones. 


\section{L'euphémisme}

41 Discours «en deçà » qui atténue l'expression de réalités choquantes ou d'interdits culturels et religieux en privilégiant des formes stéréotypées ${ }^{25}$, l'euphémisme est particulièrement apprécié. C'est le cas de hay («chose») aussi usité dans la vie courante :

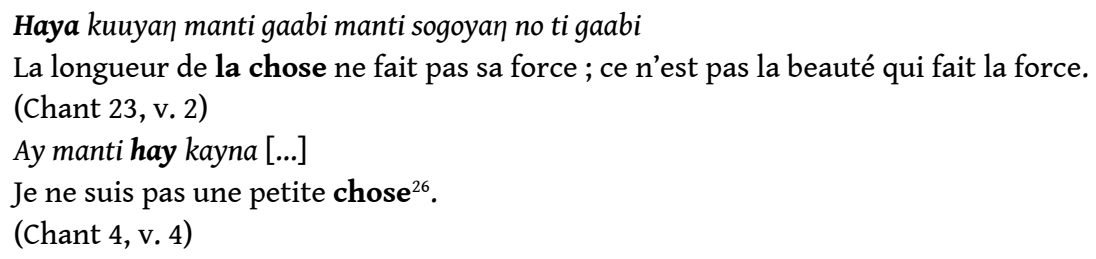

L'emploi de verbes sans connotation sexuelle comme "faire» ou "rendre service » relève également de l'euphémisme; seul le cotexte (soit l'environnement verbal de l'unité) permet d'y voir une désignation de l'acte sexuel :

Wayboro daara ciray morso / [...] / Wallaahi kala ay ma a nafa

Cette femme claire bien rouge / [...] / Au nom de Dieu je vais lui rendre service.

(Chant 19, v. 63-69)

C'est aussi le cas du verbe kaaru («monter, chevaucher »), que l'on peut employer dans un sens commun "l'enfant est monté sur l'arbre ", comme dans un sens plus ésotérique lorsqu'un génie prend possession d'une personne (on dit qu'il la chevauche); ici, l'homme « monte » ou « chevauche » la femme pour la posséder :

Hala da mayjida ${ }^{\prime 27}$ kaaru danga day a si ba suba koyne

Quand l'époux monte, c'est comme s'il n'en voulait vraiment plus le lendemain

[c'est-à-dire jusqu'à épuisement].

(Chant 3, v. 13)

De même que le verbe mooru, "caresser, flatter, presser doucement, faire une passe rituelle sur un animal» dans le septième chant, Mooru a ma koy ganda («Caresse jusqu'en bas »), bien plus que de référer à une simple caresse, renvoie à l'acte sexuel.

Parler de bas-ventre (kone) pour désigner le sexe féminin est une synecdoque qui permet à la chanteuse de réfuter d'éventuelles attaques et d'entretenir dans le même temps la connivence entre femmes mariées, qui comprennent ce langage allusif :

Kone wa kone koy wa

Gare au bas-ventre gare à la propriétaire du bas-ventre.

(Chant 24, v. 7)

L'évocation de la douceur (kaani : "douceur, plaisir ») - en lieu et place du surnom donné au sexe (natte: «foufounette ») inconnu des hommes et des vierges - apparaît aussi comme un discours «en deçà "... Par ces deux désignations, elle donne au sexe féminin une connotation doublement positive: d'une part, le surnom implique une relation de proximité et de connivence ; d'autre part, il est associé à ce qui est doux et agréable. En outre, elle permet une connivence avec les autres participantes, toutes des femmes mariées, qui - comme elle - se représentent aussitôt ce qu'évoque la soliste :

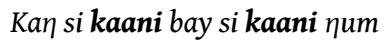

Quiconque ne connaît pas le plaisir ne lave pas le plaisir.

(Chant 1, v. 7) 


\section{L'ellipse}

47 Les omissions qui émaillent les chants, notamment dans les séquences narratives (Adam, 1992, 46), peuvent être qualifiées d'ellipses. Celles-ci attirent l'attention sur le non-dit. L'acte sexuel apparaitt par exemple, dans le premier chant, entre les deux vers décrivant le pénis avant et après l'acte :

A koy beene a ye ga ka ganda ga zaba

Il est monté, il est retombé et s'est affaissé.

(Chant 1, v. 57)

La séquence énumérative peut aussi être concernée par l'ellipse :

Ay ga ay hayni hawru bay za fu / Ay ga ay haamo hawru bay za fu / Hayni hawru mana kande ay / Kawi nda hawru mana kande ay / [...] Ay ga kawi nda hawru bay za fu / Ay ga hayni hawru bay za fu / Kaasumo mana kande ay

Je connais ma pâte de mil depuis la maison / Je connais ma pâte de sorgho depuis la maison / La pâte de mil ne m'a pas amenée / La boule de mil délayée et la pâte ne m'ont pas amenée / [...] / Je connais la boule de mil délayée et la pâte depuis la maison / Je connais la pâte de mil depuis la maison / Le mélange de feuilles vertes ne m'a pas amenée.

(Chant 7, v. 3-13)

La répétition rythme le chant, lui donne une cohérence et crée une attente dans l'auditoire. En énumérant ce qu'elle connaît déjà (soit les différents plats courants en région songhay-zarma), la locutrice souligne que ce n'est pas là la raison de son mariage et implicitement nous met sur la voie de ses véritables motivations : ce qu'elle recherche, ce qu'elle ne connaît pas encore - et qui est éludé ici - ce sont les relations sexuelles. Cette interprétation est renforcée par le lien concret qui existe entre nourriture et sexualité. La soliste s'en sert d'ailleurs pour construire sa plainte, l'énumération ne servant qu'à signifier le manque, la frustration de l'épouse négligée.

\section{La métaphore}

Comme la comparaison, la métaphore associe des objets au sexe. Mais là où la comparaison explicite l'analogie, la métaphore ne fait que la suggérer. C'est en ce sens que celle-ci est de l'ordre du caché alors que la première est de l'ordre du révélé.

Les métaphores sont nombreuses pour représenter le sexe masculin, tantôt « botte de mil» (gollo) ou de paille (baata), tantôt couteau (santeela), rasoir (siino), corne (hilli) ou arbres, quand il n'est pas pilon (hinji)... Les femmes ont tendance à moins employer de métaphores pour référer à leur propre sexe, lui préférant souvent des désignations moins imagées. Tout au plus est-il qualifié - comme nous l'avons vu précédemment - de "petit récipient» (koppiize), d'«emplacement» (gurbo) ou de "trou sans fond» (gatamba), les deux derniers référents renvoyant au fait que la jeune femme n'est plus vierge.

51 Par contre, les chanteuses semblent se délecter de métaphores toutes plus imaginatives les unes que les autres pour parler de l'acte sexuel, comme celles du coup de machette (zafa) ou de cornes (nukum), de l'acte de déchirer les feuilles de palmier (kongu san) voire du travail collectif (boogu). Or ce dernier est généralement effectué par les jeunes hommes pour aider un ami ou leurs beaux-parents dans les travaux champêtres, la construction d'un grenier ou d'une maison. On peut donc voir dans cette métaphore un rapprochement entre les relations sexuelles et une activité sociale qui permet de s'inscrire dans la communauté. 

action :

Dafe beeri cabuyan no / to / Siino na kambo di / [...] / Mooru a ma koy ganda / Sobay ga mooru a ma koy ganda / Dafe beeri cabuyan no

C'est le rasage du grand con / D'accord! / Le rasoir a écorché le côté / [...] / Caresse jusqu'en bas / Continue de caresser jusqu'en bas / C'est le rasage du grand con.

(Chant 9, v. 4-12)

L'étonnant, dans ce chant, est le télescopage entre registre vulgaire et courant, explicite et métaphorique ; la présence d'un terme grossier (dafe : «con»), qui ne se dit jamais au quotidien (il est réservé aux jurons et aux insultes), est ainsi associée à des termes relevant d'un registre ordinaire, détournés ici pour désigner métaphoriquement le sexe masculin (siino: «rasoir») ou l'acte sexuel (cabuyan: « rasage »). Sachant que l'enfant est rasé lors de son baptême, comme un nouveau chef lors de son intronisation, on pourrait penser que l'acte sexuel renvoie à quelque chose de sacré, de rituel. Mais la présence du terme dafe-comme le contexte général du chant - nous pousse plutôt à y voir une bouffonnerie où l'on ramènerait le rite au niveau d'une grivoiserie.

arfois filées, comme dans le sixième chant où la soliste se moque

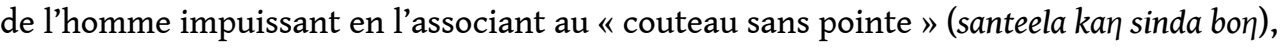

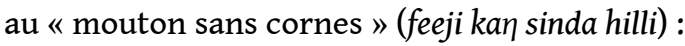

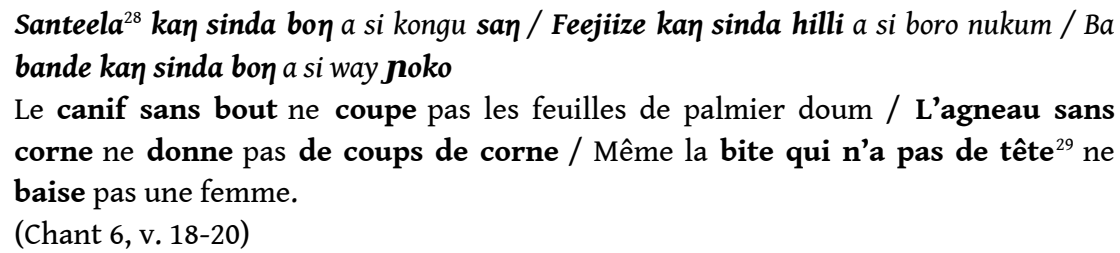

Le vers 20 donne rétroactivement, par analogie (à la fois syntaxique et métaphorique), l'interprétation des deux vers précédents. On retrouve ce même procédé dans le huitième chant :

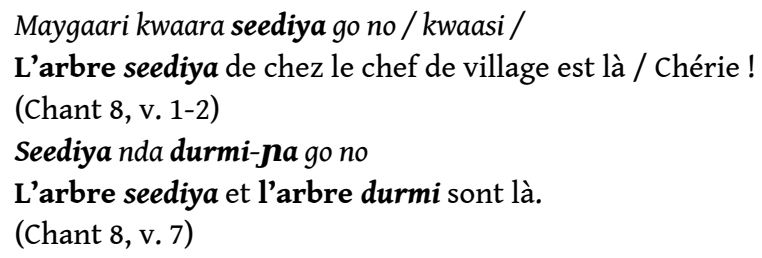
métaphoriquement au sexe de l'homme, non seulement par leur forme phallique, mais aussi parce qu'ils produisent un latex laiteux, aisément associable au sperme. Cette analyse est confirmée par le cotexte, puisque la soliste parle d'une femme qui rêve de faire l'amour avec les hommes assis dans la cour du chef de village ; seules la retiennent la honte et la crainte de tomber enceinte.

57 «Ragoût d'œufs de gecko » est une autre métaphore du sperme :

Cita gunguri sakku bande nda dafe may no ga ti koyize?

Ragoût d'œufs de gecko de la bite ou du con, qui est le prince?

(Chant 18, v. 2) 
lui est un reptile saurien sur lequel pèse un tabou alimentaire : les Songhay-Zarma ont l'interdiction d'en manger et ne doivent même pas consommer une denrée qui aurait été en contact avec lui. On retrouve ici l'association entre nourriture et sexe et le fait que les œufs de gecko comme le sperme sont tous deux liés à la reproduction renforce un peu plus cette assimilation. Que penser alors de ce sakku, plat ordinairement apprécié, accommodé pour l'occasion à des ingrédients particulièrement dégoûtants? N'y aurait-il pas un rapprochement à faire avec la fellation?

À la question de savoir qui est le « prince », les chanteuses répondent inlassablement :

Dafe day ga ti koyize kan i ga a gar ganda ga a joko kubay ra

C'est vraiment le con qui est prince, lui que l'on trouve en bas pour le baiser dans

l'obscurité.

(Chant 18, v. 3)

On peut voir, dans cette relation à deux, un jeu de synecdoques où la bite est l'homme, le con la femme; ce jeu nous donne à voir une représentation des relations de genre inversée par rapport aux représentations sociales en vigueur dans la société songhayzarma, puisque dans ce cas c'est la femme qui domine. Mais les synecdoques semblent atténuer l'inversion des rapports de domination et ne restreindre celle-ci qu'à l'activité sexuelle.

Comme le montrent ces exemples, le réseau métaphorique se développe à la fois au sein d'un chant particulier et tout au long de la performance.

\section{Les onomatopées et les idéophones}

Par l'énonciation d'onomatopées et d'idéophones, les chanteuses montrent l'intensité des ébats et reproduisent, tout en mimant l'acte sexuel, les gémissements de la femme ou les bruits de la literie sous les assauts de l'homme :

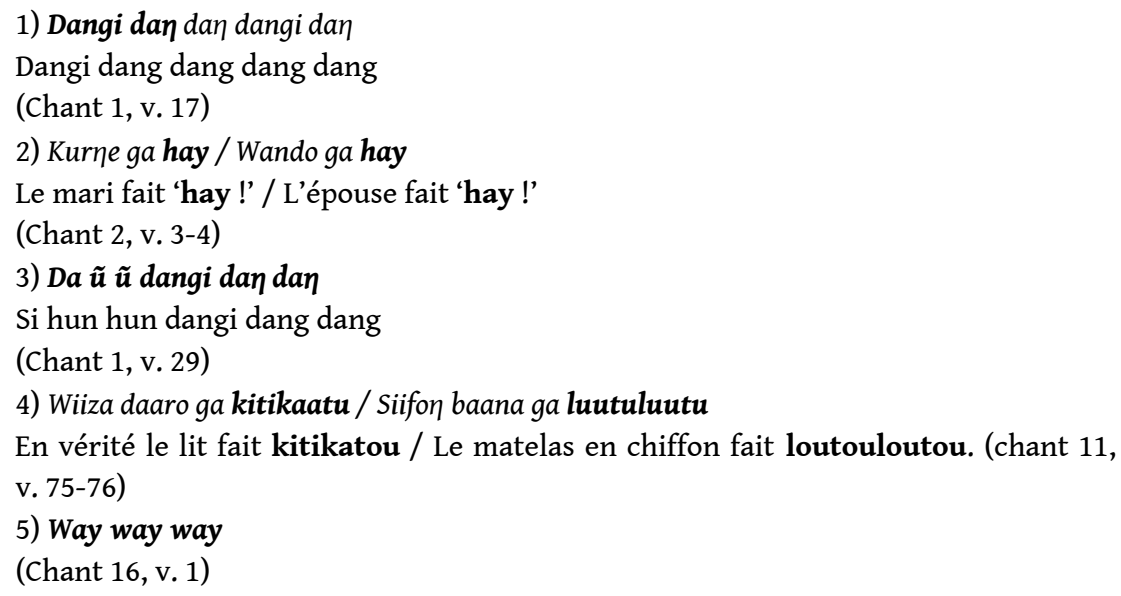

Les rires et les regards qui ponctuent ces vers marquent une connivence entre « initiées» qui comprennent toutes à quoi font référence ces onomatopées; une connivence qui s'exprime dans le chant par une opposition construite entre celles qui reconnaissent un sexe masculin et celles qui le prennent pour autre chose.

Parfois la soliste substitue le terme désignant le sexe féminin par des sons n'ayant aucun rapport avec le référent. Elle crée ainsi un sous-entendu que le cotexte et le contexte permettent aux « initiées » de saisir : 


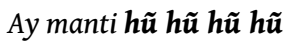

Je ne suis pas hun hun hun hun

(Chant 4, v. 6)

Revuz, 1998) qui permettent de faire exister la parole d'autrui. Le discours représenté donne aux nombreuses anecdotes et témoignages chantés un "effet de réel » et d'authenticité. C'est pourquoi les chanteuses multiplient les énoncés « polémiques » en discours représenté, ce qui leur évite d'assumer la responsabilité des propos qu'elles tiennent, en les mettant dans la bouche d'une autre. Comme lorsqu'elles font parler un marabout obsédé et adultérin :

Astangafurullay ! / Alfa / Way daara diray morso / [...] / Wallaayi kala ay ma a te / [...] / Wa sobay zankey wa sobay / [...] / Wa kuurey kambandi [...] / Wa walhey lambandi / [...] / Wallaahi kala ay ma a te /[...] / Wallaahi kala ay ma a hõ

Que Dieu me (nous) pardonne! / Marabout / Cette femme claire bien rouge / [...] / Au nom de Dieu au point que je le lui fasse ! / [...] / Continuez, enfants, continuez ! / [...] / Rangez vos peaux ! / [...] / Mettez les ardoises contre le mur ! / [...] / Au nom de Dieu au point que je le lui fasse ! / [...] / Au nom de Dieu au point 
que je la veuille.

(Chant 19, v. 1-26)

71 Le discours représenté lui permet à la fois de montrer l'hypocrisie qu'il y a pour un lettré musulman à enseigner le Coran sans en respecter les préceptes et de signaler l'obsession sexuelle de la gent masculine en général. Par l'interjection astangafurullay, placée au début du chant, la soliste se distancie des propos qu'elle rapporte et demande par avance pardon à Allah pour ceux-ci, et se montre ainsi plus respectueuse envers l'islam que le marabout dont elle parle. Mais cette imploration n'est peut-être pas uniquement celle de la soliste, car elle peut tout aussi bien relever du discours intérieur du marabout, qui s'excuserait pour ses mauvaises pensées et pour ses actes. L'ambiguïté demeure ici, alors que les autres paroles peuvent clairement être attribuées au marabout (cf. l'emploi de la première personne du singulier, mais aussi de l'impératif pluriel qui implique qu'un "je" s'adresse à ce "vous"). La polyphonie - en mêlant à sa description de l'adultère des termes arabes liés à l'islam et à la prière (astangafurullay: "Que Dieu nous pardonne» v. 1 ; laahillaaha illallaahu : «Il n'y pas d'autre divinité que Dieu ", notamment) - permet à la soliste de dire ce qui ne peut être dit tout en demandant pardon pour ce blasphème. Et ces paroles provocantes n'empêchent pas ces mêmes chanteuses d'interrompre les entretiens pour aller prier !

\section{Les énoncés gnomiques}

Les énoncés gnomiques, qui se caractérisent par l'effacement de la source énonciative, fonctionnent comme des arguments d'autorité, puisqu'ils se font l'écho d'une voix collective (Perrin, 2008) et « cette objectivation [a] comme conséquence d'offrir une polyphonie potentielle d'un discours qui semble devoir plus largement être pris en charge » (Vion, 1998, 198-199). Dans l'exemple ci-dessous, la soliste en profite pour rappeler les principes moraux en vigueur, qui font que la femme doit accepter la polygamie :

Hiijay manti taali / Faakaaray manti yanje

Le mariage n'est pas un problème / La discussion n'est pas la dispute.

(Chant 5, v. 26-27)

73 Posés comme une vérité générale incontestable, ces énoncés gnomiques ne permettent que difficilement la remise en question de leur validité ; et c'est ce qui va permettre aux énonciatrices de les utiliser pour faire passer un message tantôt moral, tantôt subversif, se ménageant dans ce dernier cas une porte de sortie («ce n'est pas moi qui le dis »). C'est le cas dans cet exemple :

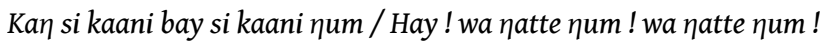

Qui ne connaît pas le plaisir ne lave pas le plaisir / Eh! Lavez foufounette! Lavez

foufounette!

(Chant 1, v. 8-9)

74 S'il marque clairement une opposition entre femmes mariées et pucelles, cet énoncé est en fait bien plus complexe. Ces deux vers se suivent et marquent par la substitution, répétée à deux reprises, de kaani ("plaisir») par natte («foufounette»), une équivalence; ce qui nous permet d'y voir une interchangeabilité et par conséquent de comprendre l'énoncé « laver le plaisir » à un autre niveau que celui de la seule hygiène intime. Ne peut-on ainsi pas y voir une apologie du plaisir solitaire? 
L'énoncé gnomique permet de transformer des paroles taboues en quelque chose de dicible, comme dans l'exemple ci-dessous, où la soliste joue sur la pudeur et le dévoilement, lorsqu'elle fait allusion à l'orgasme féminin :

Man nda man yan no a ga tu boro se ? / [...] / Ne nda ne yan no a ga tu boro se / [...] / Hanga gandey a ga tu boro se / [...] / Banda daaro ra a ga tu boro se / Boro jinde banda no a ga

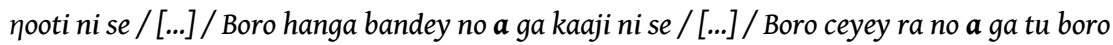
se / Boro moyey ra no a ga tu boro se

Où et où le ressent-on? / C'est ici et ici qu'on le ressent / C'est par le derrière des oreilles qu'on le ressent / [...] / C'est dans l'échine que l'on ressent / [...] / C'est dans la nuque qu'il bouge / [...] C'est derrière les oreilles qu'il la fait frissonner / [...] / C'est dans les pieds qu'on le ressent / C'est dans les yeux qu'on le ressent.

(Chant 1, v.23-48)

Parfois, malgré l'effacement de l'énonciateur, certaines traces énonciatives persistent. C'est le cas lorsqu'une expression implique une connotation péjorative comme la "chienne de Damana », qui laisse transparaître à travers la disqualification une forme de subjectivité :

Daamana hansi si haawi bay Daamana hansi si haawi bay

La chienne de Damana ne connaît pas la honte, la chienne de Damana ne connaît pas la honte.

(Chant 5, v. 2)

77 Cet amalgame entre une femme du village zarma de Damana (Zarmaganda) et un animal, déjà dépréciatif en soi, est renforcé par le fait qu'il s'agit d'un chien, espèce particulièrement méprisée en pays musulman. Cette association montre ainsi un désaccord et un mépris profond pour le comportement de cette femme, et marque de fait une prise en charge énonciative.

Si l'on pensait les chanteuses parties dans le registre subversif, elles ponctuent par-ci par-là leurs chants de préceptes moraux comme l'abstinence avant le mariage :

Boro ma zalay ka hiiji a day ga ba boro ma zalay ka gunde sambu

Il vaut mieux, pour une femme, être courtisée avant d'être mariée qu'être enceinte au cours de causeries.

(Chant 23, v. 13)

La formulation impersonnelle - typique de l'énoncé gnomique - efface toute trace énonciative, et il est d'autant plus difficile de savoir qui parle que le vers précédent et le suivant sont empruntés à un genre différent, les chants de jeunes filles non mariées chantés sur la place de fête ou lors des mariages :

Hay boro ma ni niya dima fun a day ga ba boro ma kubay nda gunde

Eh! il vaut mieux trouer le lit de sa mère plutôt que d'être mariée enceinte ${ }^{31}$.

(Chant 23, v. 14)

\section{Entre procédures d'ancrage énonciatif et de débrayage...}

Le chant racontant l'anecdote d'un homme qui commet l'adultère avec l'épouse de son fils parti en migration saisonnière est un bel exemple des stratégies énonciatives que la soliste met en place, alternant embrayages (ou ancrages) et débrayages, au point que l'on ne sache plus qui parle.

81 Pour ce faire, elle joue sur trois niveaux: la situation de communication (soit le marcanda), la situation d'énonciation (notamment les embrayeurs) et l'histoire racontée. Dans un premier temps, elle entame son chant par une salutation: 
Beebe baaba Udu baaba / Kala baani walla

Père de Bébé, père de Oudou / Rien que la santé.

(Chant 11, v. 1-2)

82 En contexte de marcanda, cette salutation s'avère fictive, car les hommes sont absents. Par contre, du point de vue de la situation d'énonciation, elle introduit le père comme allocutaire et ce faisant crée une ambiguïté sur son propre rôle d'énonciatricelocutrice. Tout est donc fait pour créer un effet de réel (apostrophe et réponse). Toutefois la réponse, donnée par la soliste elle-même et sans imiter la voix masculine, pose clairement un décalage entre situation de communication et situation d'énonciation.

83 La soliste poursuit ce dialogue fictif avec le père et en profite pour se distinguer d'une deuxième protagoniste de l'anecdote, la belle-fille prénommée Hanay. Signalons que celle-ci est clairement définie comme un tiers dont on parle, mais qui ne s'exprime pas :

Si haaru ay se si mo kar ay se / Ay manti Haanay

Ne me souris pas, ne me fais pas des clins d'œil ! / Je ne suis pas Hanay.

(Chant 11, v. 4-5) l'apostrophe et de la deuxième personne du singulier. Cette absence de «je » fait partie d'une stratégie d'insinuation qui va se poursuivre par le passage au récit à la troisième personne. En effet, alors que nous nous attendions à ce qu'elle s'adresse à Oudou comme dans les répliques précédentes, l'énonciatrice se repositionne et raconte un récit à la troisième personne, où Oudou n'est plus en position de locuteur ni d'allocutaire :

Hano kaך Udu fun Kumaasi ${ }^{32}$ haawi ga banda haawi ga jine / Wiiza daaro ga kiitikaatu

Le jour où Oudou est revenu de Kumasi, la honte était derrière, la honte était devant / Car le lit faisait 'kitikatou'.

(Chant 11, v. 74-75)

87

\section{l'énonciation.}

Cette stratégie est renforcée par l'introduction de discours représentés au sein du récit, clairement pris en charge cette fois par les locuteurs du récit. Aussi la soliste cite-t-elle les paroles du beau-père et en relève-t-elle toute la lubricité, lorsqu'il épie sa belle-fille en train de se laver :

A ne manti ay hinne no na a zonkom / I'nda ay caley no / A ga ciray a ga loy-loy / Wala

faani gaasu

Il a dit : «Je n'étais pas le seul à épier / J'étais avec mes camarades / C'est rouge,

c'est brillant / Comme une calebasse de crème ».

(Chant 10, v. 27-30) 
Comme l'énonciatrice n'a plus besoin d'assumer la responsabilité des propos qu'elle fait tenir aux personnages, elle se laisse aller à une certaine liberté de ton.

\section{Quels sont les sens et fonctions de ces chants?}

On peut dégager quatre fonctions principales de ces chants et des procédés discursifs mis en place: un exutoire à la colère, la constitution d'une connivence entre «initiées", la revendication d'une plus grande équité des rapports de genre et la constitution d'une identité féminine.

\section{Exutoire à la situation sociale qu'elles vivent}

Ces chants ont une fonction d'exutoire ou de «soupape, canalisant précisément l'agression latente sous-jacente à toute soumission à une autorité » (Larguèche, 1997, 122). Dans le cas du marcanda, la soumission est double : à l'homme d'une part et à la situation polygame d'autre part. L'exutoire passe alors par la moquerie, l'inversion des valeurs et l'expression de frustrations.

Si, dans certains chants, les chanteuses critiquent les femmes incapables de résister à un homme, le plus souvent, c'est à ce dernier qu'elles s'attaquent, ou à la morale sociale qui leur impose la sujétion à ce dernier. C'est ainsi qu'elles se moquent de deux catégories opposées de « mâles »: ceux qui ne peuvent résister à la tentation du sexe et les impuissants qui ne peuvent remplir leur devoir conjugal, comme ici :

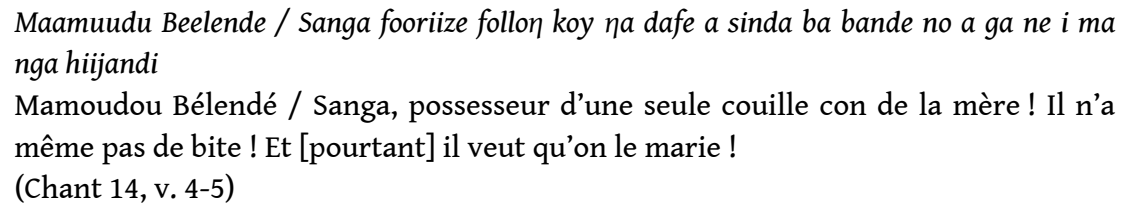

En révélant l'infirmité de cet homme, l'énonciatrice-locutrice le ridiculise et affiche son mépris envers celui qui ne pourra ni procréer ni donner de plaisir à une femme. L'exaspération est mise en évidence par le juron "con de la mère!», que je n'ai quasiment jamais entendu dans la bouche d'adultes d'origine libre ${ }^{33} ;$ si cette velléité de mariage soulève une telle indignation, c'est d'abord par l'égoïsme aveugle qui la motive sans que soient pris en compte les besoins de celle qui sera choisie.

Les chanteuses poussent parfois la provocation encore plus loin et mettent en scène une toute-puissance féminine, alors même que celle à qui ces chants sont adressés vit une situation de soumission absolue, elle qui doit accepter en silence l'arrivée d'une coépouse. Cette toute-puissance apparaît dans une parole en apparence libérée de tout tabou. Libérée à l'instar de la prostituée qu'une soliste convoque comme locutrice, pour faire étalage de tous les bénéfices qu'elle retire de la débauche: la fortune et l'indépendance. En valorisant sa profession, pourtant socialement réprouvée, elle s'oppose à celles que la morale sociale valorise habituellement (les femmes mariées, mères d'une nombreuse progéniture) à qui elle demande de reconnaître sa réussite :

Wa ay no kobi saatarey wa ay no kobi

Femmes mariées, applaudissez-moi, applaudissez-moi !

(Chant 6, v. 17)

Ay hanna ga sinji no / I ma ay dafayizo kar ga say / Mooto beerey kan ni ga di / Zuzuki

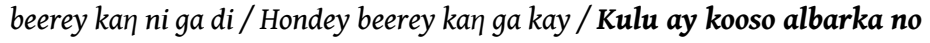


Je passerai la nuit plantée [dehors] / Pour qu'on éparpille mon berlingot / Ces grandes voitures que tu vois / Ces grandes Suzuki que tu vois / Ces grandes Honda garées / Toutes sont la bénédiction de ma chatte.

(Chant 12, v. 6-11) place dans un rapport de force avec son fiancé. Chez les Songhay-Zarma, la morale veut qu'une fille arrive vierge au mariage, et si d'aventure - ce n'est pas le cas, le mariage est annulé et la famille de la jeune fille se couvre de honte, à moins que les époux ne s'arrangent en secret. Dans ce chant, l'interdit dépassé n'est pas tu, mais assumé par la locutrice-jeune fille. Par les synecdoques employées (cf. « emplacement » et «trou sans fond »), elle réduit son identité à un vagin défloré. Mais loin de disparaitre sous la honte, elle exige le mariage, tout en respectant la hiérarchie sociale (cf. l'emploi de la forme particulière d'injonction ( $\mathrm{ma}$ ) qui marque une déférence) :

Hiija hane ma ta ni gurbo ma ta ni gatamba ga a ganday

Le jour du mariage prends ton emplacement prends ton trou sans fond et enlacele.

(Chant 17, v. 4)

Cette revendication d'une équité passe parfois par l'expression des besoins sexuels de la femme. C'est le cas quand le chant se présente comme une fable où la femme, désignée par une synecdoque - le con -, reçoit de son époux toute une série de cadeaux qu'elle refuse, à l'exception du pénis de son mari. La gradation dans la série narrative crée une tension et conduit progressivement l'épisode à son point culminant, soit l'acceptation joyeuse du pénis par la vulve :

I na dafe no yo dafe ne nga si ba / I na dafe no haw dafe ne nga si ba / [...] / I na dafe no gooro a ne nga si ba / I na dafe no hincin i na a no hincin mo a ne nga si ba / I na dafe no bande / A haaru ga ta

On a donné un chameau au con, le con a dit qu'il n'en voulait pas / On a donné une vache au con, il a dit qu'il n'en voulait pas / [...] / On a donné de la cola au con, il a dit qu'il n'en voulait pas / On a aussi donné une chèvre au con il a dit qu'il n'en 
voulait pas / On a donné une bite au con / Il a ri et a accepté ${ }^{34}$.

(Chant 10, v. 3-10) chanteuses et les autres participantes ont à briser explicitement ensemble les tabous liés à la sexualité. Ces énoncés attirent en effet "par leur aspect d'interdit et rendent tout-puissant celui qui les utilise puisqu'il transgresse alors l'interdit » (Larguèche, 1997, 126). Ce plaisir n'est pas seulement verbal, mais s'exprime aussi dans et par les danses : c'est par exemple le cas quand elles miment l'acte sexuel, c'est le cas également quand elles représentent le sexe de l'homme en érection en plaçant un bâton sous leur pagne. conjurer le malheur, en faisant de leur condition de coépouse une identité revendiquée, une force, une solidarité, qu'elles partagent. Et le sentiment de pouvoir que génèrent ces chants particuliers peut être interprété comme une manière de se réapproprier un destin qui ne leur appartient pas, même si, au bout du compte, elles retrouveront leur époux et leurs coépouses, quoiqu'elles fassent. 


\section{Vers la constitution d'une identité féminine}

106 Toute prise de parole, comme l'écrit Amossy $(1999,9)$, « implique la construction d'une image de soi ». Or les chants sont collectifs, dans leur énonciation présente, mais aussi dans le temps, puisqu'ils appartiennent à un répertoire de littérature orale chanté par plusieurs générations de femmes. On peut donc dire qu'ils participent à la construction d'une identité féminine, et plus précisément, d'une identité de femmes mariées.

Cette image collective se déploie à la fois par l'énonciation d'une parole subjective (cf. l'emploi du «je » ou du «nous») et d'énoncés sentencieux qui présentent comme vérité générale un discours transgressif au regard de la société songhay-zarma. Cette image collective se construit aussi par une opération de « déstéréotypage ». Au modèle de la femme soumise, devant accepter la polygamie, mais aussi les besoins sexuels de son mari, les chanteuses opposent l'impuissance masculine, la domination du vagin sur la verge et la revendication de leurs propres besoins sexuels. Et si, au quotidien, la sexualité a comme but la procréation, dans ces chants, aucune mention n'en est faite et seul le plaisir est évoqué. L'oscillation constante entre explicite et implicite s'explique par le fait que ces représentations de la sexualité féminine sont en porte à faux avec la morale que véhicule la société dans laquelle les chanteuses évoluent.

Mais il ne faut pas voir ici qu'un message subversif. L'image de soi collective que créent les chants est traversée par des représentations fluctuantes: les chanteuses jouent à cache-cache avec des opinions, se les réapproprient pour les contredire aussitôt. Ainsi, après avoir revendiqué un certain pouvoir et des besoins propres, elles conseillent à la femme dont l'époux se remarie d'accepter la situation nouvelle qui lui est imposée et de la voir comme un bienfait :

Warjida $^{35}$ goro ni bon ga / Hiijay manti taali / Faakaaray manti yanje / [...] / Ni bonkaanay ga kand'a / [...] / Ni kayno no ga ka ya / Ni wayco no ga ka ya / [...] / Ni duura no ga kand'a $/[. .$.

Grande épouse, assieds-toi correctement ! / Le mariage n'est pas un malheur / La causerie n'est pas une bagarre / [...] / C'est ta chance qui l'a amenée / [...] / C'est ta petite sœur qui arrive / C'est ta coépouse qui arrive / [...] / C'est ta richesse qui l'a amenée.

(Chant 5, v. 25-40)

\section{Conclusion}

Je t'avoue que je ne pouvais pas penser que de tels mots pouvaient sortir de la bouche d'une Zarma, car je pense que s'il y a bien un peuple pudique, ce sont eux, non?

C'est ainsi qu'une amie zarma, aujourd'hui émigrée à Paris, mais ayant grandi au Niger, a réagi à l'écoute de ces chants ${ }^{36}$. Cette réaction me parait être une parfaite illustration du propos de cet article. Car, arrivée célibataire en France, elle a été tenue à l'écart de ces moments d'intimité entre femmes mariées où l'on parle de la sexualité de manière crue et où l'on se moque de ce que l'on ne peut évoquer publiquement.

$110 \mathrm{Au}$ cours de ce rituel particulier, qu'est le marcanda, les femmes font des joutes verbales d'insultes et chantent la colère, la douleur, la tristesse puis l'acceptation de ne plus être la dernière épousée. Mais les chants peuvent être véritablement impudiques, voire obscènes s'ils sont chantés par des femmes d'origine captive entretenant une relation complice (malgré leur différence de " classe ») avec la femme pour laquelle est organisé 
le rite et en fonction de la liberté accordée par le mari. La situation de communication est donc un des premiers éléments qui permettent de contourner l'interdit, le tabou langagier.

111 La parole se fait transgressive non seulement par ce qu'elle dit sur la sexualité, mais aussi sur les rapports de genre. Pour autant, y a-t-il revendication? Car, si « revendiquer », selon le Petit Robert, c'est « réclamer une chose sur laquelle on a un droit [...] et, par extension, assumer pleinement », les chanteuses chantent, non pas pour obtenir des droits, mais pour permettre à celle qui perd son statut de dernière épousée d'accepter ce changement de situation. S'inscrivant dans ce processus de deuil, le renversement des représentations des rapports de genre n'est donc que transitoire.

Seul compte alors le pouvoir de chanter - en l'absence des hommes - ce qu'il n'est pas possible d'exprimer en d'autres occasions; il y a ainsi jouissance dans l'expression de cette parole libérée des tabous imposés par une société patrivirilocale. Si l'homme dont les chanteuses se moquent plutôt crûment est absent, c'est pour mieux s'adresser à la femme dont le conjoint se marie à nouveau, qui peut ainsi atténuer sa douleur en riant de ses futures rivales, mais aussi de cet époux qui - pourtant - les lui impose.

Cette transgression n'est, en outre, tolérée que parce que la parole est déléguée à des femmes d'origine captive et en particulier garaasa ("artisan du cuir ») réputées pour leur maitrise dans ce domaine. En effet, il est inenvisageable - pour des femmes nobles - de se montrer aussi impudiques, car c'est la pudeur, précisément, qui les distingue des captives. Tout au plus osent-elles chanter « qui connait le bonheur lave le bonheur " sans y associer explicitement le surnom du sexe. Le processus rituel passe ainsi nécessairement, pour les femmes d'origine libre, par la médiation de chanteuses ayant un statut inférieur au leur. En exprimant tout haut ce que les femmes libres pensent tout bas, les chanteuses permettent aux membres de l'auditoire de se défouler sans risquer l'opprobre public, et à celle pour qui est organisé le marcanda d'entamer un processus d'acceptation de sa nouvelle situation.

114 Pour évoquer la sexualité habituellement taboue, les chanteuses alternent provocation et évitement, paroles explicites et implicites. L'explicite apparaît à la fois dans la situation de communication et dans le registre discursif. Mais, même dans une situation de communication "artificielle", les chanteuses gardent une part d'implicite et exploitent celle-ci pour créer une connivence avec celles qui sont à la fois leurs proches, mais aussi potentiellement leurs rivales (quatre des six chanteuses du corpus présenté ici ont le même époux !). De cette relation entre les chanteuses et l'auditoire naît une complicité entre femmes mariées ; une connivence construite, tout au long des chants, sur la base de ce qui les oppose aux hommes, mais aussi aux jeunes filles, les deux sources de leur malheur.

115 Au-delà de ce discours sur la sexualité, les nombreuses synecdoques nous amènent à penser qu'il cache en réalité un propos plus général sur les relations de genre. Sans doute peut-on en déduire que, si la sexualité est taboue, elle l'est bien moins pourtant que l'évocation des rapports de genre et du pouvoir que la femme possèderait malgré tout. Mais quoiqu'il arrive, à la fin du rituel, le rideau tombe et la réalité reprend ses droits : à l'arrivée du mari, les chanteuses se taisent... puis vont le saluer, ainsi que ses femmes, avant de prendre congé. 


\section{BIBLIOGRAPHIE}

ADAM, Jean-Michel, 1992, Les textes : types et prototypes. Récit, description, argumentation, explication et dialogue, Paris, Nathan Université.

ADAM, Jean-Michel, 1999, Linguistique textuelle. Des genres de discours aux textes, Paris, Nathan Université.

AMOSsY, Ruth, 1999, La notion d'ethos de la réthorique à l'analyse du discours, in R. Amossy (éd.), Images de soi dans le discours. La construction de l'ethos, Lausanne, Ed. Delachaux \& Niestlé, p. 9-30. AUTHIEZ-REVUZ, Jacqueline, 1998, Énonciation, méta-énonciation. Hétérogénéités énonciatives et problématiques du sujet, in R. Vion (éd.), Les sujets et leurs discours. Énonciation et interaction, Aixen-Provence, Université de Provence, p. 63-89.

BARTHES, Roland, 1968, L'Effet de réel, Communications, $\mathrm{n}^{\circ}$ 11, p. 84-89.

BERNARD, Yves \& WHITE-KABA, Mary, 1994, Dictionnaire zarma-français, Paris, Niamey, ACCT.

BONHOMME, Marc, 1998, Les figures clés du discours, Paris, Seuil.

BORNAND, Sandra, 2002, Insultes rituelles entre coépouses. Étude du marcanda (Zarma, Niger), ethnographiques.org, $\mathrm{n}^{\circ}$ 7, avril 2005.

BORNAND, Sandra, 2010, Chants de douleur des femmes zarma, in Florence Dupont, Maria Manca, Claude Calame, Bernard Lortat-Jacob (dir.), La Voix Actée. Pour une nouvelle ethnopoétique, Paris, Kimé, p. 159-173.

BORNAND, Sandra, 2012, Voix de femmes songhay-zarma du Niger, Cahiers « Mondes anciens » [en ligne] 3.

CALAME, Claude, 2000, Le Récit en Grèce Ancienne, Paris, Belin.

Charaudeau, Patrick, maingueneau, Dominique (dir.), 2002, Dictionnaire d'analyse du discours, Paris, Seuil.

DIARRA Fatoumata-Agnès, 1971, Femmes africaines en devenir. Les femmes zarma du Niger, Paris, Ed. Anthropos.

KERBRAT-ORECCHIONI, 1998, L'implicite, Paris, Armand Colin [1re éd., 1986].

LARGUÈCHE, Évelyne, 1983, L'effet injure, Paris, PUF.

LARGUÈCHE, Évelyne, 1997, Injure et sexualité. Le corps du délit, Paris, PUF.

LEGUY, Cécile, 2001, Le proverbe chez les Bwa du Mali, Paris, Karthala.

OLIVIER DE SARDAN, Jean-Pierre, 1984, Les sociétés songhay-zarma (Niger, Mali) : chefs, guerriers, esclaves, paysans, Paris, Karthala.

PERRIN, Laurent, 2008, Le sens montré n'est pas dit, in M. Birkelund, M.-B. Mosegaard Hansen et C. Norén (éds), L'énonciation dans tous ses états : mélanges offerts à Henning Nølke, Bern, Berlin, Bruxelles, Frankfurt am Main, New York, Oxford, Wien, Ed. Peter Lang, coll. « Foreign Language Study », p. 157-187.

TERSIS, Nicole, 1981, Économie d'un système. Unités et relations syntaxiques en zarma (Niger), Paris, SELAF. 
VION, Robert, 1998, Du sujet en linguistique, in Robert Vion (éd.), Les sujets et leurs discours. Énonciation et interaction, Aix-en-Provence, Université de Provence, p. 189-202.

VION, Robert, 2006, Modalisation, dialogisme et polyphonie, in Laurent Perrin (dir.), Le sens et ses voix. Dialogisme et polyphonie en langue et en discours, Recherches linguistiques, $n^{\circ} 28$, Université Paul Verlaine Metz, p. 105-125.

\section{NOTES}

1. La région songhay-zarma se trouve à l'Ouest du Niger. Les Songhay sont aussi présents au Mali, alors que les Zarma habitent uniquement le Niger. Si les membres de leur aristocratie revendiquent un ancêtre différent, la langue et le patrimoine socioculturel mettent en évidence une certaine unité de l'ensemble songhay-zarma. La langue songhay-zarma est définie comme une langue à tons par les différents chercheurs qui s'y sont intéressés (Tersis, 1981) - toute voyelle est prononcée avec une certaine hauteur mélodique de la voix (deux tons simples : haut et bas; deux tons modulés : ascendant et descendant). Cependant, à l'exception de quelques termes, les tons ne sont pas indispensables à la compréhension; c'est pourquoi l'orthographe songhay-zarma utilisée dans cet article (tout comme celle employée par le service d'alphabétisation) ne les prend pas en compte.

2. S'il n'y a pas d'ambiguïté pour deux femmes d'un même ménage, lorsqu'elles sont trois, la deuxième choisit son camp, alors qu'avec quatre femmes, les deux premières sont "grandes ", les deux dernières « petites ".

3. Sur les sept chanteuses, trois viennent de cantons zarma (Hamdallaye, Tondikiwindi et Liboré) et quatre du canton songhay de Gotheye. Quatre d'entre elles originaires de différents lieux sont coépouses, deux sont leurs amies, et la troisième - fait étrange (Bornand, 2012) - est la nièce utérine de l'une d'entre elles. Sa présence s'explique par sa maîtrise des chants et par le fait qu'elle les accompagne très souvent aux mariages.

4. Je reprends ici la distinction signalée par Calame $(2000,20)$ entre «la situation "réelle", "référentielle" de communication de la parole, avec les paramètres d'ordre social et psychologique qui en déterminent la figure » et « la situation d'énonciation telle qu'elle paraît, par la médiation de la langue, dans l'énoncé qui est lui-même l'objet du processus de communication ».

5. Concept polysémique désignant tant la honte que la pudeur et impliquant un certain idéal de bienséance et de savoir-vivre.

6. Ceci serait également le cas dans une situation rituelle.

7. Par exemple, fuyize (litt. "petite maison») désigne la chambre, buutalize (litt. "petite bouteille ») le flacon, boosayize le fruit du tamarinier.

8. Bernard et White-Kaba (1994) citent dabiinayize pour désigner le clitoris. Le terme combine le suffixe-ize (petit, enfant) et le terme de base (dabiina) désignant la datte.

9. Hormis dans ces chants, je n'ai que très rarement entendu un adulte utiliser ces termes. Les enfants et adolescents (jamais des filles) quand ils injurient, le font très discrètement, signifiant le plus souvent - par un geste de la main (main ouverte, paume tendue vers la personne que l'on veut insulter). La force de l'injure demeure toutefois, puisque cela entraînait aussitôt des bagarres entre les enfants.

10. Dans le sens de l'ensemble du sexe féminin.

11. Variante kourtey (elle-même variante du songhay) de duwa « l'avoir ».

12. Ou bagu-bagu selon les prononciations.

13. Variante songhay du terme zarma roogo « manioc ».

14. Instrument en bois pour remuer la sauce. 
15. Quand la chanteuse dit A jeeneewa, elle fait un mouvement de bras : avec sa main elle forme un demi-cercle de droite à gauche, puis accélère lorsqu'elle fait remonter son bras et sa main (la paume tournée vers le haut) comme si elle dessinait avec ce geste le redressement. L'expression $A$ jeeneewa n'a pas de signification particulière mais renvoie à ce geste ; c'est pour cela que je l'ai traduite par «se mettre en érection ».

16. Ceci montre la souplesse du sexe masculin et sa virilité.

17. Baata : herbe. Aristida longiflora : chaume.

18. Liw-liw est un idéophone qui représente « un redressement subit, vigoureux ».

19. «La dimension culturelle de l'individu, comme du discours, conduit les sujets à reformuler des propos existants, à utiliser, sans même nécessairement le savoir, du déjà-dit, du "prêt à porter" discursif, à mettre en scène des opinions dont ils ne sont pas les énonciateurs originels. Dans ces conditions quand un locuteur parle, il n'y a pas que lui dans sa propre parole, mais ça parle à travers lui. » (Vion, 1998, 195).

20. Ici, c'est un démonstratif à valeur déictique [ne wo : ici / dém.].

21. Il n'y a qu'à voir sa fonction syntaxique.

22. Si Diarra (1971, 64-68) évoque un «forgeron-griot [qui] parle de l'acte sexuel et de ses préliminaires de telle sorte que les jeunes gens et jeunes filles se trouvent informés avant la consommation du mariage» (64), je n'ai eu aucune confirmation sur la poursuite de telles pratiques dans les années 2000. Aucune des femmes ne m'a parlé de cette transmission par le « forgeron » et moi-même je n'y ai jamais assisté malgré des séjours en région zarma depuis 1994. Lors des mes enquêtes en région zarma sur les questions sexuelles (2004-2005), la plupart des femmes me disaient ne pas avertir leurs filles (soit parce que «cela ne se faisait pas », soit parce qu' « en ville il n'y en avait plus besoin »). Seules des femmes d'origine captive m'ont dit avertir leur fille qu'il fallait qu'elle se montre réticente lorsque le mari s'approchait et qu'elle lave son sexe.

23. La migration saisonnière est une pratique courante chez les hommes songhay-zarma, qui partent en dehors de la saison des cultures travailler dans les pays voisins, afin de gagner de l'argent pour leur famille.

24. Cet aspect ne sera pas développé ici, car je n'ai pu filmer la séance.

25. Figure dérivée, il peut recouvrir des tropes tels que la métaphore, ou des figures syntaxiques telles que la périphrase ou l'ellipse.

26. On retrouve ici les caractéristiques valorisées lorsqu'il y a nomination (longueur, grandeur).

27. Terme hausa désignant l'homme marié.

28. Santeela : petit couteau qu'utilisent les femmes songhay-zarma en travaillant les feuilles de palmier doum pour en faire des nattes.

29. Soit qui ne bande pas.

30. L'emploi du terme «énonciateur » varie selon les chercheurs (cf. notamment Charaudeau et Maingueneau, 2002). Pour ma part, suivant en cela Calame (2000), je distingue l'énonciateur, qui s'adresse à un ou plusieurs énonciataire(s) lors de l'acte effectif de production de l'énoncé, du locuteur et de l'allocutaire qui sont les actants inscrits dans le texte.

31. «Trouer le lit de sa mère » renvoie à l'affaissement du matelas tant la fille dort dessus. Cette fille est alors celle qui ne sort pas et ne fréquente pas les hommes avant son mariage.

32. Le Ghana est un lieu d'exode traditionnel des Songhay-Zarma.

33. J'ai entendu ces insultes le plus souvent - quoique très rarement - dans la bouche de personnes d'origine captive ou du jasare (« griot généalogiste et historien »).

34. On pourrait interpréter cette synecdoque comme représentative du fait que la femme est d'abord perçue comme un organe sexuel et reproducteur. Mais il me semble que ce n'est pas ici le cas : d'une part, parce que la femme n'est pas la seule à être, dans ce chant, désignée par une synecdoque, l'homme l'est aussi; d'autre part, parce qu'aucun chant de ce corpus n'évoque l'enfantement, alors que les chants de marcanda qui ne sont pas obscènes l'évoquent. 
35. Le terme warjida est emprunté au hausa. L'expression songhay-zarma est wande beeri (litt. « grande épouse »).

36. Je remercie d'ailleurs Safia Amadou pour son aide dans la transcription et la traduction de ces chants.

\section{RÉSUMÉS}

Chez les Songhay-Zarma du Niger se déroule, durant les mariages polygames, une cérémonie particulière, le marcanda, au cours de laquelle des femmes d'origine captive évoquent, en chansons et en l'absence des hommes, la sexualité, ses plaisirs et ses déviances. Ces énonciatrices particulières abordent, dans leurs chants, des sujets tabous et semblent prendre systématiquement à contre-pied les valeurs prônées par la société.

Maniant à la fois parole directe (emploi de termes vulgaires) et indirecte (métaphores, jeux sur les voix énonciatives, etc.), les chanteuses créent une complicité avec les femmes de l'auditoire, qui entendent - à travers les voix de captives - une parole qu'elles ne pourraient prononcer. Car les seules chanteuses autorisées à chanter ces chants se situent doublement au bas de l'échelle sociale: en tant que femmes et en tant que descendantes de captives qui - dit-on et contrairement aux êtres d'origine libre - «ne connaissent pas la pudeur ».

Among the Songhay-Zarma of Niger a particular ceremony known as the marcanda, takes place during polygamous marriages. During this ceremony, women who descended from war captives, evoke, in song and in the absence of a male presence, sexuality, its pleasures and its deviances. In their songs, these particular "speakers" confront taboo subjects and seem to systematically oppose the dominant social values. Versed in both direct (use of vulgar terms) and indirect discourse (metaphors, play on the voices, etc.), the singers establish a kind of complicity with the women in the audience who hear-through the voices of war captives-a set of words they would never be able to utter. Indeed, the only people allowed to sing these songs are doubly at the bottom of the social ladder: as women and as descendants of war captives who, in contrast to freemen,-it is said-“do not know sexual modesty".

\section{INDEX}

nomsmotscles Songhay, Zarma

Thèmes : anthropologie (Afrique)

Index géographique : Niger

Keywords : War Captives, Explicit, Genre, Implicit, Niger, Performance, Polygamy, Pragmatic, Sexuality, Songhay, Zarma, Song

Mots-clés : chant, explicite, genre, implicite, performance, polygamie, pragmatique, sexualité 